\title{
ERRATA
}

\section{Erratum: Do excited states exist in a system of two neutrons? [JETP Lett. 67, No. 11, 903-909 (10 June 1998)]}

\author{
D. V. Aleksandrov, E. Yu. Nikol'skiĪ, B. G. NovatskiĪ, and D. N. Stepanov
}

Kurchatov Institute Russian Science Center, 123182 Moscow, Russia

\author{
R. Wolski \\ Institute of Nuclear Physics, 31342 Kraków, Poland
}

[S0021-3640(98)01816-7]

PACS numbers: 13.75.Cs, 99.10. $+\mathrm{g}$

This article consists of an error which was reported by the authors. Namely, the term "deuteron" was erroneously used throughout the article instead of a correct term "dineutron." Therefore, below we offer a complete reprint of the article with the correction being incorporated. We apologize for the error.

\section{Do excited states exist in a system of two neutrons?}

D. V. Aleksandrov, E. Yu. Nikol'skiǏ, B. G. NovatskiǏ, and D. N. Stepanov

Kurchatov Institute Russian Science Center, 123182 Moscow, Russia

\section{R. Wolski}

Institute of Nuclear Physics, 31342 Kraków, Poland

(Submitted 23 April 1998)

Pis'ma Zh. Éksp. Teor. Fiz. 67, No. 11, 860-865 (10 June 1998)

The interaction in a system of two neutrons $(2 n)$ in the reaction $\mathrm{T}(d$, $\left.{ }^{3} \mathrm{He}\right)$ at energy $E_{d}=31 \mathrm{MeV}$ is investigated experimentally. Nuclearunstable ${ }^{2} n$, with a decay width $\Gamma=(1.1 \pm 0.2) \mathrm{MeV}$, is observed with a large transverse cross sections in the interval of angles from $6^{\circ}$ to $13^{\circ}$ $\left(d \sigma / d \Omega\left(6^{\circ}\right) \sim 10 \mathrm{mb} / \mathrm{sr}\right.$ in the center-of-mass system). Two wide energy peaks were observed in the ${ }^{3} \mathrm{He}$ spectra. It is proposed that broad resonances with energies $E^{*}=(3.6 \pm 0.3)$ and $E^{*} \sim 11.8 \mathrm{MeV}$, populated in the reaction $\mathrm{T}\left(d,{ }^{3} \mathrm{He}\right)$, are excited in the $n-n$ system. Their energy positions satisfy the interval rule $E_{4}: E_{2} \cong 3.3$, indicating the possible existence of a "rotational band" with the characteristics $2^{+}$ and $4^{+}$. The "radius" of the dineutron is estimated from the relation 


$$
\begin{aligned}
& \Delta E=\hbar^{2} l(l+1) / 2 \mu R^{2} \text { to be } \sim 8 \mathrm{fm} \text {. (C) } 1998 \text { American Institute of } \\
& \text { Physics. [S0021-3640(98)00511-8] }
\end{aligned}
$$

PACS numbers: 13.75.Cs

The question of the forces acting between two particles is a fundamental problem of classical and quantum physics. The solution of this problem has made it possible to understand the nature of electromagnetic and gravitational forces. It is well known that the existence of numerous excited states in the hydrogen atom was the key to the construction of the "planetary" model of the Bohr atom. Nuclear forces differ sharply from electromagnetic forces by their very short range, and the deuteron - the simplest nuclear system with an anomalously low binding energy $(\epsilon=2.22 \mathrm{MeV})-$ does not have excited bound states like the hydrogen atom.

However, the experimental study of $p n$ and $p p$ scattering has firmly established an interaction in the final state in these systems with isospin $T=1$ and it has been shown that singlet $d$ and $2 p$ systems possess virtual levels near the decay threshold. In the 1960s and 1970s the hypothesis of the charge independence and symmetry of nucleon-nucleon interactions was checked experimentally in measurements of the scattering lengths. A small difference was found in the $n-n$ and $p-n$ interactions: $a_{n n}=-16.6 \mathrm{fm}$ and $a_{n p}$ $=-23.7 \mathrm{fm}$ (see, for example, the review in Ref. 1). In contrast to $p-p$ and $p-n$ scattering, because of the unavailability of neutron targets the main means of studying the $n-n$ interaction have been the reactions $\mathrm{D}(n, p), \mathrm{T}(n, d), \mathrm{D}(d, 2 p), \mathrm{T}\left(d,{ }^{3} \mathrm{He}\right)$, and $\mathrm{T}\left(t,{ }^{4} \mathrm{He}\right)$. We note that in these investigations emphasis has been placed on confirmation of the hypothesis of charge independence and symmetry of the nuclear forces and not on a search for excited states of unstable ${ }^{2} n$. As a rule, the spectra have been obtained in a narrow energy range near zero binding energy of the dineutron. ${ }^{2}$ On the other hand, in high-energy physics the searches have been conducted for "narrow" dibaryonic resonances $\left({ }^{2} p\right)$ with energies of tens and even hundreds of $\mathrm{MeV}$, which are traditionally far from the excitation energies characteristic for low-energy nuclear physics. ${ }^{3}$

The objective of the present work was to search for excited states of the dineutron in the reaction $\mathrm{T}\left(d,{ }^{3} \mathrm{He}\right)$ by the conventional methods of nuclear spectroscopy in the interval from the ${ }^{2} n$ ground state up to the maximum possible energies admissible under the experimental conditions $\left(E^{*} \sim 15 \mathrm{MeV}\right)$. Of the three variants of the search for dibaryonic resonances $(2 n, 2 p, n p)$ the first one was chosen because a purely nuclear interaction of two identical particles occurs in this case.

The work was performed on the cyclotron at the Kurchatov Institute Russian Science Center, at a maximum deuteron beam energy $E=31 \mathrm{MeV}$. The spectra of ${ }^{3} \mathrm{He}$ from the reaction $\mathrm{T}\left(d,{ }^{3} \mathrm{He}\right)$ were measured in the range of angles $6-13^{\circ}$. This imposed a strict limit on the intensity of the incident beam because of the enormous background due to elastically scattered deuterons. The average current of the deuterium ions on the targets was equal to $\sim 0.01 \mu \mathrm{A}$.

Two self-supporting titanium foils of the same thickness $5.1 \mathrm{mg} / \mathrm{cm}^{2}$, one of which 
was saturated with tritium, served as targets. The tritium content in the Ti-T target and the presence of impurities in it were determined by special cyclotron measurements of the elastic scattering of deuterons with $E=13 \mathrm{MeV}$. The cross sections for the elastic scattering of deuterons by tritium were taken from Ref. 4 . The measurements established the content of tritium in the irradiated Ti-T foil to be $38.4 \%$ (in terms of the number of atoms relative to $\mathrm{Ti}$ ).

The reaction products were detected with a telescope of semiconductor silicon counters with thicknesses $30 \mu \mathrm{m}$ ( $\Delta E$ - ionization-loss detector) and $1.2 \mathrm{~mm}$ ( $E-$ total-absorption detector), connected to a spectrometric system for multivariate analysis. The solid angle of the detecting system was equal to $1.3 \times 10^{-4} \mathrm{sr}$.

The spectra of the ${ }^{3} \mathrm{He}$ nuclei were measured in the energy range 5-35 MeV with good statistical accuracy. In addition, spectra were systematically taken on Ti-T and $\mathrm{Ti}$ targets for each angle. The reaction ${ }^{48} \mathrm{Ti}\left(d,{ }^{3} \mathrm{He}\right)$ and the reaction of the recoil nucleus ${ }^{3} \mathrm{He}\left(d,{ }^{3} \mathrm{He}\right) \mathrm{D}$ on the trace impurity ${ }^{3} \mathrm{He}(4-5 \%)$ in the Ti-T target (the peak in the discrete part of the spectra near $29 \mathrm{MeV}$ ) served as benchmarks for the energy calibration. The presence of this quantity of ${ }^{3} \mathrm{He}$ nuclei is due to the $\beta$ decay of tritium. Figure 1 shows the energy spectra of helium nuclei from the reaction $\mathrm{T}\left(d,{ }^{3} \mathrm{He}\right)$ after subtraction of the impurity from the reaction $\left(d,{ }^{3} \mathrm{He}\right)$ on titanium, the cross sections on which were found to be an order of magnitude smaller than on tritium. The spectra were measured at angles of $6,8,10$, and $13^{\circ}$ in the laboratory coordinate system. An intense peak corresponding to the interaction of two neutrons in the final state is clearly seen in the hard part of the spectra. In the figure it is marked by an arrow labeled with ${ }^{2} n$, and in the text below we shall refer to it as the ground state of the dineutron. A wide bump centered near $23 \mathrm{MeV}$ is observed in the "soft" part of the spectra to the left of the ground state. A subsequent analysis - approximation of the bump by a Gaussian distribution - established that as the measurement angle increases, the center of the bump shifts continuously in the direction of low energies. This shift corresponds to the well-known energy-angle kinematic dependence for a binary reaction, which can be explained by the presence of a wide resonance in the nuclear system ${ }^{2} n$. Besides the features indicated, a continuous distribution with a maximum near $14 \mathrm{MeV}$, whose relative contribution increases rapidly with angle and becomes dominant at $13^{\circ}$ (Fig. 1d), is present in the entire measured energy range. A similar picture has been observed before in measurements of the spectra of the reaction $\mathrm{T}\left(d,{ }^{3} \mathrm{He}\right)$ with $11 \mathrm{MeV}$ deuterons. ${ }^{5}$

We endeavored to explain the complicated structure of the spectra obtained by the well-known mechanisms of interactions in very light nuclei.

1. Resonance-like structures due to different two-step processes can appear in the spectra of the products of nuclear reactions on light nuclei. The probability of a two-stage mechanism of the reaction $\mathrm{D}+\mathrm{T}$ depends on the kinematic conditions of the experiment, and it is impossible to rule out a priori the appearance of wide maxima in the spectrum of ${ }^{3} \mathrm{He}$ in the reaction channel $\mathrm{D}+\mathrm{T} \rightarrow n+{ }^{4} \mathrm{He}^{*}$ with the formation of the excited levels of the ${ }^{4} \mathrm{He}$ nucleus and their subsequent decay ${ }^{4} \mathrm{He}^{*} \rightarrow{ }^{3} \mathrm{He}+n$. Monte Carlo simulation of this process, taking into account the excitation of the levels $E^{*}=21,21.8$, and 23.3 $\mathrm{MeV}$ in the ${ }^{4} \mathrm{He}$ nucleus, ${ }^{6}$ established that the energies and widths of the observed maxima do not conform to the theoretical curves - dotted lines in Fig. 2 (the computed curves have not been normalized to the experimental data). 

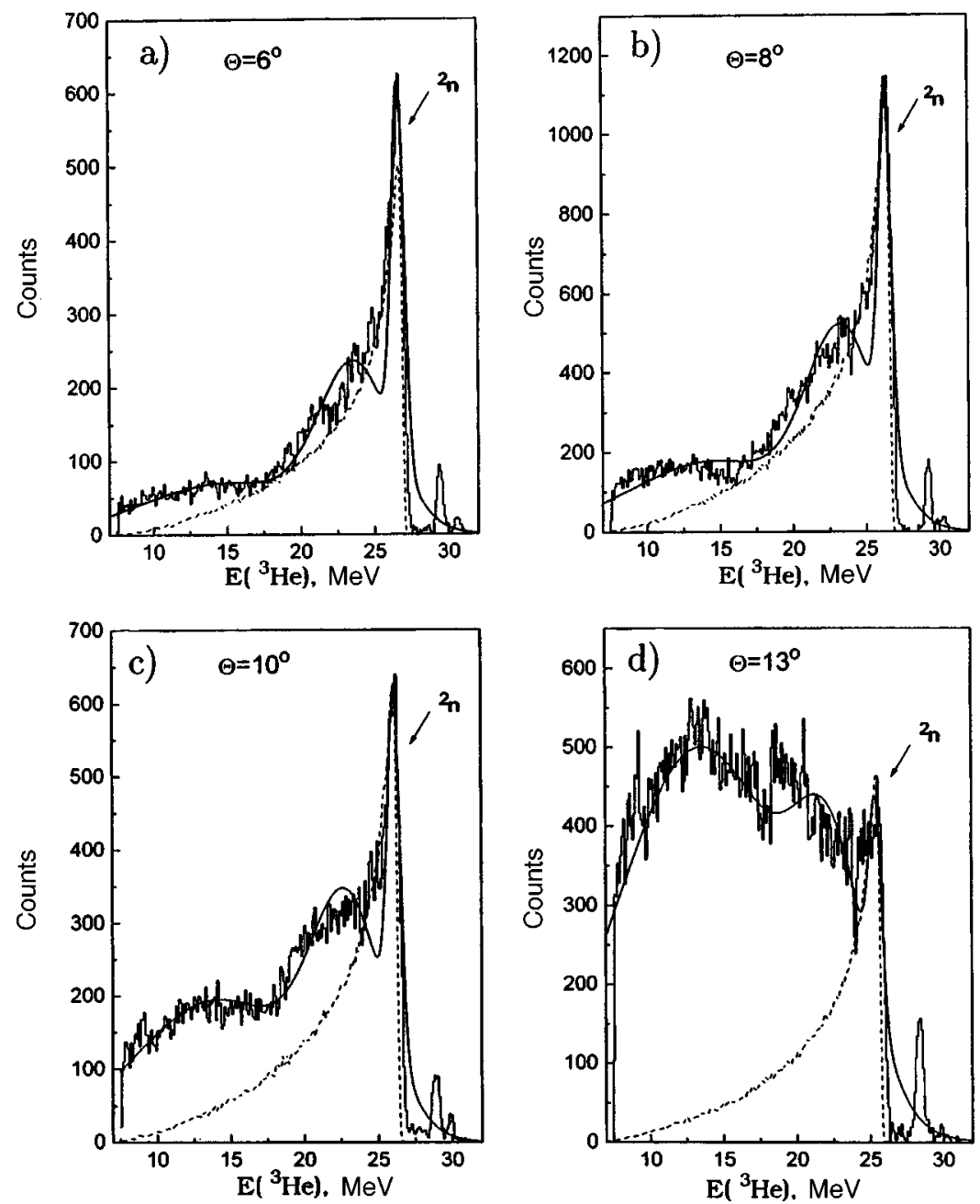

FIG. 1. Energy spectra of ${ }^{3} \mathrm{He}$ ions from the reaction $\mathrm{T}\left(d,{ }^{3} \mathrm{He}\right) 2 n$ at angles of $6-13^{\circ}$ in the laboratory system. The dotted lines show the calculation according to the Migdal-Watson theory with $a_{n n}=-16 \mathrm{fm}$. The solid curves show the results of a fit by three Gaussian normal distributions.

2. The continuous distributions at all measured angles were calculated in the Migdal-Watson (MW) approximation. Figure 1 (dotted lines) shows the computed curves for scattering length $a_{n n}=-16 \mathrm{fm}$. Satisfactory agreement was obtained between the computed and experimental distributions only for high energies. At $13^{\circ}$ only the sharp rise in the hard part of the spectrum could be described by the MW method.

3. The characteristic features of the measured spectra also cannot be reproduced by calculations of the three-particle phase distribution for $\mathrm{D}+\mathrm{T} \rightarrow{ }^{3} \mathrm{He}+n+n$ (solid line in Fig. 2).

Thus none of the processes considered above reproduced the continuous ${ }^{3} \mathrm{He}$ distributions. We propose the hypothesis that in the reaction $\mathrm{T}\left(d,{ }^{3} \mathrm{He}\right)$, besides the dineutron 


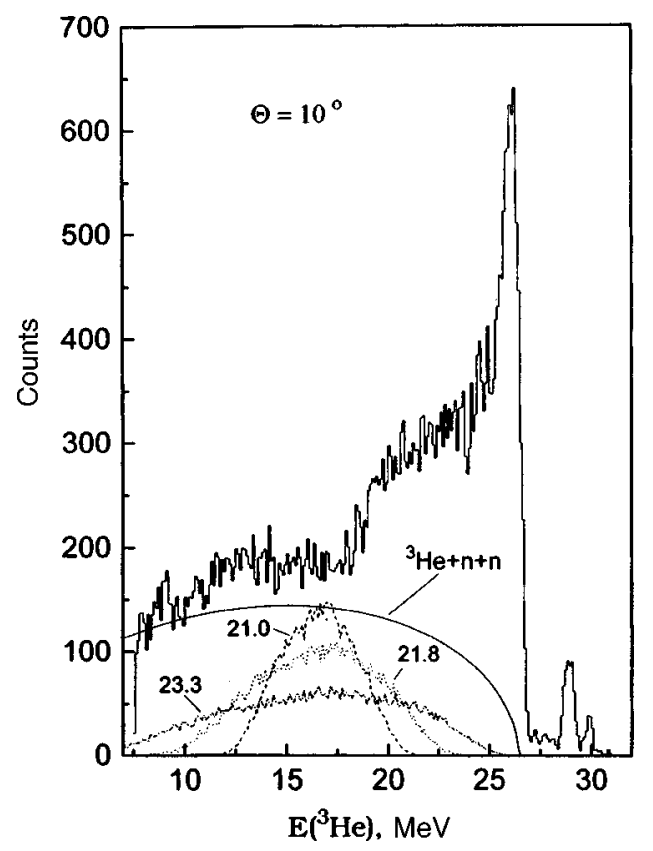

FIG. 2. Energy spectrum of ${ }^{3} \mathrm{He}$ nuclei from the reaction $\mathrm{T}\left(d,{ }^{3} \mathrm{He}\right) 2 n$ measured at an angle of $10^{\circ}$. The solid curve corresponds to the three-particle phase volume of the system ${ }^{3} \mathrm{He}+n+n$ in the outgoing channel. The dotted lines show the computational results for the process $\mathrm{T}+\mathrm{D} \rightarrow n+{ }^{4} \mathrm{He}^{*} \rightarrow{ }^{3} \mathrm{He}+n+n$, which proceeds via ${ }^{4} \mathrm{He}$ levels with $E^{*}=21.0,21.8$, and $23.3 \mathrm{MeV}$.

ground state, two wide resonances associated with excitation of the $n-n$ system are populated. To check this conjecture, a computer was used to carry out a $\chi^{2}$-minimizing fit of the continuous spectra by three Gaussians, with automatic variation of the positions, widths, and areas of the desired resonances. The computational results are shown in Fig. 1 (solid lines). Satisfactory agreement was obtained between the experimental and computed distributions. After switching to the center-of-mass system, the excitation energies of the resonances were determined as $E^{*}=(3.6 \pm 0.3) \mathrm{MeV}$ and $E^{*} \sim 11.8 \mathrm{MeV}$ relative to the ${ }^{2} n$ ground state. The values agree with one another, to within the error limits, at all measured angles. The energies satisfy the well-known interval rule $E_{4}: E_{2} \cong 11.8: 3.6$ $=3.3$, indicating the possible existence of "rotational" excited states of the dineutron with quantum characteristics $2^{+}$and $4^{+}$. The values of the spin and parity of the resonance $E^{*}=3.6 \mathrm{MeV}$ in ${ }^{2} n$ are also consistent with the systematics of all even-even nuclei, according to which their first levels, except for the magic levels, possess $2^{+}$ characteristics. The "radius" of a dineutron was estimated from the moment of inertia of the $n-n$ system $\Delta E=\hbar^{2} l(l+1) / 2 \mu R^{2}$ to be $\sim 8 \mathrm{fm}$. This value is close to the values of the radii of the valence neutrons in weakly bound neutron-rich nuclei, such as $\left({ }^{11} \mathrm{Li}\right.$, $\left.{ }^{11} \mathrm{Be}\right){ }^{7,8}$

In summary, in the present work we have apparently observed wide resonances in ${ }^{2} n$. We note a number of favorable factors and conditions which have led to the results obtained.

I. The choice of the $n-n$ system was a decisive factor for the search for dibaryonic 


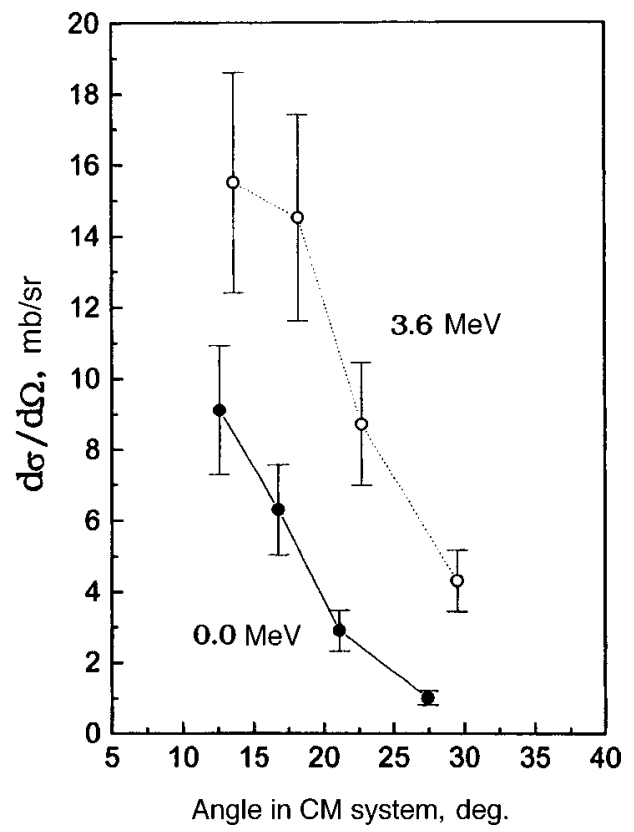

FIG. 3. Experimental angular distributions of ${ }^{3} \mathrm{He}$ from the reaction $\mathrm{T}\left(d,{ }^{3} \mathrm{He}\right)$ for the ${ }^{2} n$ ground state and a resonance with $E=3.6 \mathrm{MeV}$.

resonances: a) In $p-p$ interactions, besides nuclear forces, it is also necessary to take account of Coulomb forces and their interference effects. Since the desired resonances lie in the continuum, the Coulomb repulsion of the two protons should result in smearing of their decay widths $(\Gamma \gg 1 \mathrm{MeV})$. As an example, we indicate the unstable $2^{+}$levels of the nearest isobar-analog nuclei with $T=1:{ }^{6} \mathrm{He},{ }^{6} \mathrm{Li}$, and ${ }^{6} \mathrm{Be}$, whose widths equal $\Gamma=0.113 \mathrm{MeV}, 0.540 \mathrm{MeV}$, and $1.16 \mathrm{MeV}$, respectively. ${ }^{9}$ b) In $n-p$ scattering and in reactions where a singlet deuteron is investigated according to the spectra of the accompanying particles the analysis is greatly complicated by the contribution of the isobaranalog interaction with $=T=0$. Therefore, on account of the statistical factor, the cross section $\sigma_{s}$ for $n-p$ scattering in the singlet state appears in the formula for the total cross section with a coefficient $1 / 4$. c) In ${ }^{2} n$ a purely nuclear interaction of identical particles appears and the interpretation of the levels in $n-n$ is much simpler than in the case of $n-p$ and $p-p$ systems.

II. We note an advantage of the reaction $\mathrm{T}\left(d,{ }^{3} \mathrm{He}\right)$ for observing resonances in the dineutron. The reaction mechanism is very simple - a direct one-nucleon pickup reaction. This is indicated by the large values of the transverse cross sections $\left(d \sigma / d \Omega\left(6^{\circ}\right)\right.$ $\sim 10 \mathrm{mb} / \mathrm{sr}$ in the center-of-mass system) and their sharp forward elongation. This is evident from Fig. 3, where the experimental angular distributions for the reaction $\mathrm{T}(d$, ${ }^{3} \mathrm{He}$ ) are presented. The filled circles indicate the differential cross sections for the ${ }^{2} n$ ground state, while the open circles correspond to the resonance with $E=3.6 \mathrm{MeV}$. Conversely, the parasitic process $\mathrm{T}\left(d,{ }^{4} \mathrm{He}^{*}\right)$, proceeding with formation of unstable levels of ${ }^{4} \mathrm{He}^{*}$ and their subsequent dissociation into ${ }^{3} \mathrm{He}+n$, is more complicated (twonucleon pickup reaction). Moreover, as one can see from the table of the decay widths 
TABLE I.

\begin{tabular}{llc}
\hline \hline$E^{*}(\mathrm{MeV})$ & $J^{\pi}$ & $\Gamma(\mathrm{MeV})$ \\
0.0 & $0^{+}$ & $1.1 \pm 0.2$ \\
$3.6 \pm 0.3$ & $2^{+}$ & $5.6 \pm 0.5$ \\
$\sim 11.8$ & $4^{+}$ & $\geqslant 11$ \\
& & \\
\hline \hline
\end{tabular}

presented in Ref. 6 , the dissociation of ${ }^{4} \mathrm{He}^{*}$ proceeds predominantly with the emergence of a triton and not a ${ }^{3} \mathrm{He}$ nucleus.

The main results of this work are presented in Table I, where the excitation energies of the hypothetical resonances in ${ }^{2} n$ are given in the first column, the proposed values of the spins and parities are given in the second column, and estimates of their decay widths are given in the third column. Nonetheless, we believe that additional experiments at high incident deuteron energies $(50-60 \mathrm{MeV})$ must be performed in order to confirm the results obtained.

We thank M. V. Zhukov and I. M. Pavlichenkov for helpful discussions and a number of valuable remarks, as well as SKTB ÉP of the Ukrainian National Academy of Sciences for preparing the titanium-tritium targets.

This work was supported by Russian Fund for Fundamental Research under Grant 96-02-17298a.

${ }^{1}$ B. Kühn, Fiz. Élem. Chastits At. Yadra, 378 (1975) [Sov. J. Part. Nucl. 6(2), 139 (1976)].

${ }^{2}$ E. Baumgartner, H. E. Conzett, E. Shield, and R. J. Slobodrian, Phys. Rev. Lett. 16, 105 (1966).

${ }^{3}$ Yu. A. Troyan, A. V. Nikitin, V. N. Pechenov et al., Preprint R1-90-78, JINR, Dubna, 1990; Yad. Fiz. 54, 1301 (1991) [Sov. J. Nucl. Phys. 54, 792 (1991)].

${ }^{4}$ M. Ivanovich, P. G. Joung, and G. G. Ohlsen, Nucl. Phys. A 110, 441 (1968).

${ }^{5}$ H. T. Larson, A. D. Bacher, K. Nagatini, and T. A. Tombrello, Nucl. Phys. A 149, 161 (1970).

${ }^{6}$ D. R. Tilley, H. R. Weller, and G. M. Hale, Nucl. Phys. A 541, 19 (1992).

${ }^{7}$ P. G. Hansen, A. S. Jensen, and B. Jonson, Annu. Rev. Nucl. Part. Sci. 45, 591 (1995).

${ }^{8}$ I. Tanihata, J. Phys. G. Nucl. Part. Phys. 22, 157 (1996).

${ }^{9}$ F. Ajzenberg-Selove, Nucl. Phys. A 413, 1 (1984).

Translated by M. E. Alferieff 